\title{
BCL11A overexpression predicts survival and relapse in non-small cell lung cancer and is modulated by microRNA-30a and gene amplification
}

Ben-yuan Jiang ${ }^{1,3+}$, Xu-chao Zhang ${ }^{1,2 \dagger}$, Jian Su ${ }^{1,2}$, Wei Meng ${ }^{1,2}$, Xue-ning Yang ${ }^{1}$, Jin-ji Yang ${ }^{1}$, Qing Zhou ${ }^{1}$, Zhi-yong Chen ${ }^{1}$, Zhi-hong Chen ${ }^{1,2}$, Zhi Xie ${ }^{1,2}$, Shi-liang Chen ${ }^{1,2}$ and Yi-long Wu ${ }^{1 *}$

\begin{abstract}
Background: Aberrant activation of the proto-oncogene B-cell lymphoma/leukemia 11A (BCL11A) has been implicated in the pathogenesis of leukemia and lymphoma. However, the clinical significance of BCL11A in non-small cell lung cancer (NSCLC) remains unknown.
\end{abstract}

Results: We examined BCL11A expression at the protein and mRNA levels in a cohort $(n=114)$ of NSCLC patients and assessed the relationship between BCL11A expression and clinicopathological parameters. Data from array-based Comparative Genomic Hybridization $(\mathrm{aCGH})$ and microRNA transfection experiments were integrated to explore the potential mechanisms of abnormal BCL11A activation in NSCLC. Compared to adjacent non-cancerous lung tissues, BCL11A expression levels were specifically upregulated in NSCLC tissues at both the mRNA $(t=9.81$, $P<0.001)$ and protein levels. BCL11A protein levels were higher in patients with squamous histology $\left(X^{2}=15.81\right.$, $P=0.001)$, smokers $\left(X^{2}=8.92, P=0.004\right)$, patients with no lymph node involvement $\left(X^{2}=5.14, P=0.029\right)$, and patients with early stage disease $\left(X^{2}=3.91, P=0.048\right)$. A multivariate analysis demonstrated that in early stage NSCLC (IA-IIB), BCL11A was not only an independent prognostic factor for disease-free survival (hazards ratio [HR] 0.24 , $95 \%$ confidence interval $[\mathrm{Cl}] 0.12-0.50, P<0.001)$, but also for overall survival $(\mathrm{HR}=0.23,95 \% \mathrm{Cl} 0.09-0.61, P=$ 0.003). The average BCL11A expression level was much higher in SCC samples with amplifications than in those without amplifications ( $t=3.30, P=0.023$ ). Assessing functionality via an in vitro luciferase reporter system and western blotting, we found that the BCL11A protein was a target of miR-30a.

Conclusions: Our results demonstrated that proto-oncogene BCL11A activation induced by miR-30a and gene amplification may be a potential diagnostic and prognostic biomarker for effective management of this disease.

Keywords: BCL11A, Proto-oncogene, Non-small cell lung cancer, microRNA, Prognosis

\section{Background}

Lung cancer, especially non-small cell lung cancer (NSCLC), is the leading cause of cancer-associated deaths in China and worldwide [1]. Despite advances in early diagnosis and standard treatment, the prognosis of NSCLC patients remains poor, with 5-year survival less than $15 \%$ [2]. A better understanding of the molecular

\footnotetext{
* Correspondence: syylwu@live.cn

'Equal contributors

'Guangdong Lung Cancer Institute, 106 Zhongshan Er Rd, Guangzhou 510080, China

Full list of author information is available at the end of the article
}

mechanisms underlying lung cancer is needed for more efficient cancer management [1,3]. Molecular epidemiological studies have provided evidence that multiple mechanisms, including activation of oncogenes (EGFR, KRAS), inactivation of tumor suppressors (TP53), and dysregulation of DNA repair genes (CHEK1), contribute to early stage lung cancer development $[1,4,5]$; however, these molecular alterations are not enough to explain the heterogeneity of NSCLC. Identification of relevant molecular alterations occurring at early stage disease has the potential to identify diagnostic or predictive 
biomarkers and may provide a strategy for clinically tailored treatments to decrease the overall mortality of this disease.

The B-cell lymphoma/leukemia 11A (BCL11A) gene encodes a Krüppel zinc-finger transcription factor, which has been shown to be essential for pre-B-cell development, thymocyte maturation, and globin switching [6,7]. This gene was first identified in a rare $t(2 ; 14)(\mathrm{p} 16 ; \mathrm{q} 32.3)$ translocation in aggressive B-cell chronic lymphocytic leukemia and was regarded as a candidate oncogene, as it was often co-amplified with the REL proto-oncogene in non-Hodgkin's lymphoma and in classical Hodgkin's lymphoma $[8,9]$. Furthermore, retroviral integration into the $B C L 11 A$ locus has been shown to activate its expression, transform NIH $3 \mathrm{~T} 3$ cells, and induce myeloid leukemia in mice $[6,10]$.

BCL11A involvement in solid tumors has been rarely reported, and whether deregulation of BCL11A expression occurs in NSCLC remains unclear. To address BCL11A expression and its potential clinical relevance in detail, we determined BCL11A expression at both the mRNA and protein levels and determined its prognostic significance by correlating BCL11A expression with clinicopathologic features and survival in NSCLC patients. We also investigated the mechanisms underlying BCL11A activation in NSCLC.

\section{Results}

$B C L 11 A$ mRNA is specifically upregulated in NSCLC tissues Based on our gene expression profiling data, we found that $B C L 11 A$ was differentially expressed between cancer and adjacent non-cancerous tissues, with 3.06-fold upregulation in cancer tissues $(t=9.81, P<0.001)$. Whether comparing between all cancer and adjacent tissues or just between paired cancerous and non-cancerous tissues, BCL11A mRNA was upregulated in cancer tissues (Figure 1A, B). Furthermore, when BCL11A expression was analysed according to different histological subtypes, BCL11A mRNA was upregulated mainly in squamous cell carcinoma (SCC) (Figure 1C, D). A correlation analysis was performed to explore whether BCL11A mRNA levels were related to clinicopathological variables in patients with NSCLC. BCL11A mRNA levels had no relationship with age, sex, or histology, but they did correlate with lymph node status and disease stage. Decreased BCL11A mRNA levels were associated with lymph node involvement $\left(X^{2}=6.17, P=0.013\right)$ and advanced stage $\left(X^{2}=4.21, P=0.040\right)$ (Table 1$)$.

\section{BCL11A protein is specifically upregulated in NSCLC tissues}

Expression of BCL11A at the protein level was also investigated in 113 NSCLC and 25 adjacent non-cancerous lung tissues by immunohistochemistry. BCL11A protein staining was not found in the 25 adjacent lung tissues (Figure 2). Statistical analysis revealed no significant correlations between BCL11A protein level and age, sex, or performance status. However, BCL11A expression level was strongly associated with histology $\left(X^{2}=15.81, P=0.001\right)$, smoking status $\left(X^{2}=8.92, P=0.004\right)$, lymph node status $\left(\chi^{2}=5.14, P=0.029\right)$, and disease stage $\left(\chi^{2}=3.91, P=\right.$ 0.048) (Table 1).

BCL11A protein level correlates with disease-free survival (DFS) and overall survival (OS) in early stage squamous carcinoma NSCLC patients

For all 113 patients subjected to immunohistochemical staining for BCL11A, 68 (59.2\%) had low BCL11A expression and 45 (40.8\%) had high BCL11A expression.

High BCL11A expression was predictive of better overall survival (OS) $\left(\log \operatorname{rank} \chi^{2}=3.79, P=0.05\right)$ and better disease-free survival (DFS) (log rank $\chi^{2}=12.70$, $P=0.0004$ ) (Figure $3 \mathrm{~A}, \mathrm{~B}$ ). The median DFS of patients with low BCL11A expression levels was $23.0 \pm 4.5$ months, but in the high BCL11A expression group, only $32.6 \%$ of patients relapsed at the endpoint of follow-up. The multivariate survival analysis indicated that BCL11A expression level was an independent marker of DFS in patients with NSCLC (hazards ratio [HR] 0.40, 95\% confidence interval $[\mathrm{CI}] 0.22-0.72, P=0.002$ ). Furthermore, we found that in a subgroup of patients with early stage cancer (IA-IIB) (Table 2), and in particular early SCC (Table 3), BCL11A was not only a significant prognostic factor for DFS (HR 0.18, 95\% CI 0.05-0.66, $P=0.01$ ), but also for OS (HR 0.17, 95\% CI 0.06-0.50, $P=0.001$ ) (Figure 3C-F). No correlations between BCL11A expression and DFS or OS were observed in advanced stage (IIIA-IV) NSCLC patients (Additional file 1: Figure S1).

\section{$B C L 11 A$ gene copy number gains in lung squamous carcinoma}

Ten probes targeting $B C L 11 A$ on the Agilent aCGH chip (A_16_P15674055, A_14_P129011, A_16_P15674243, A_ 16_P00386603, A_16_P00386649 and A_14_P106886) were used to determine the normalized logarithmic intensity of BCL11A copy number changes. The average intensity of the 10 probes for BCL11A in SCC was 0.35 , significantly higher than that in adenocarcinoma $(0.03)$ $(t=5.37, P=0.0007)$. Log intensities $>0.30$ were considered indicative of gene amplification in aCGH analysis. Thus, BCL11A was amplified in SCC, while in large-cell carcinoma $(\mathrm{LCC})(\mathrm{n}=11)$ the intensity was 0.23 , indicating no $B C L 11 A$ gene amplification (Figure 4A). A significantly higher average expression level was observed for BCL11A in the SCC samples with amplifications as compared to SCC samples without amplifications $(\mathrm{t}=3.30, P=0.023)$ (Figure 4B). 


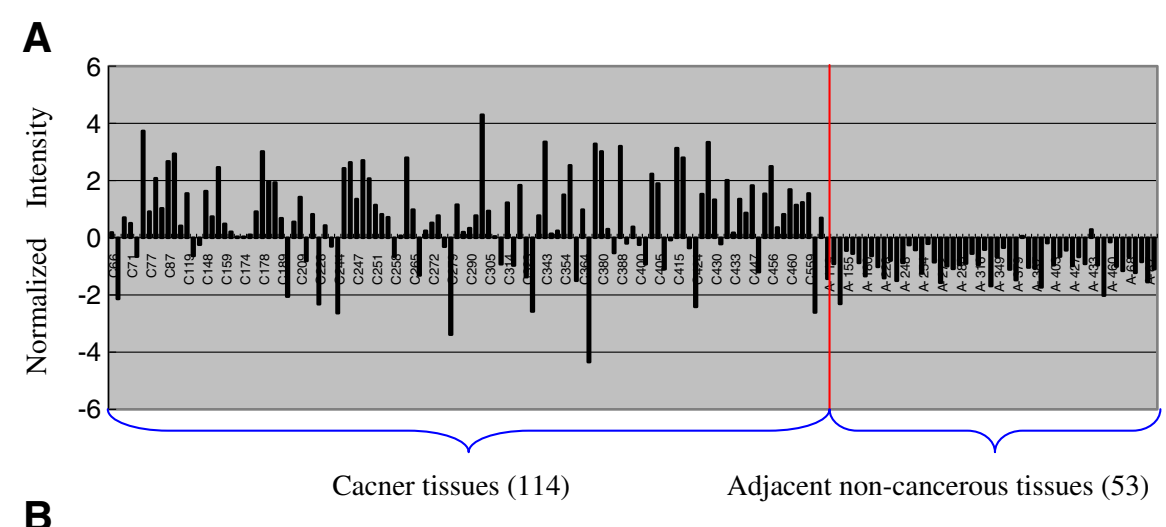

B
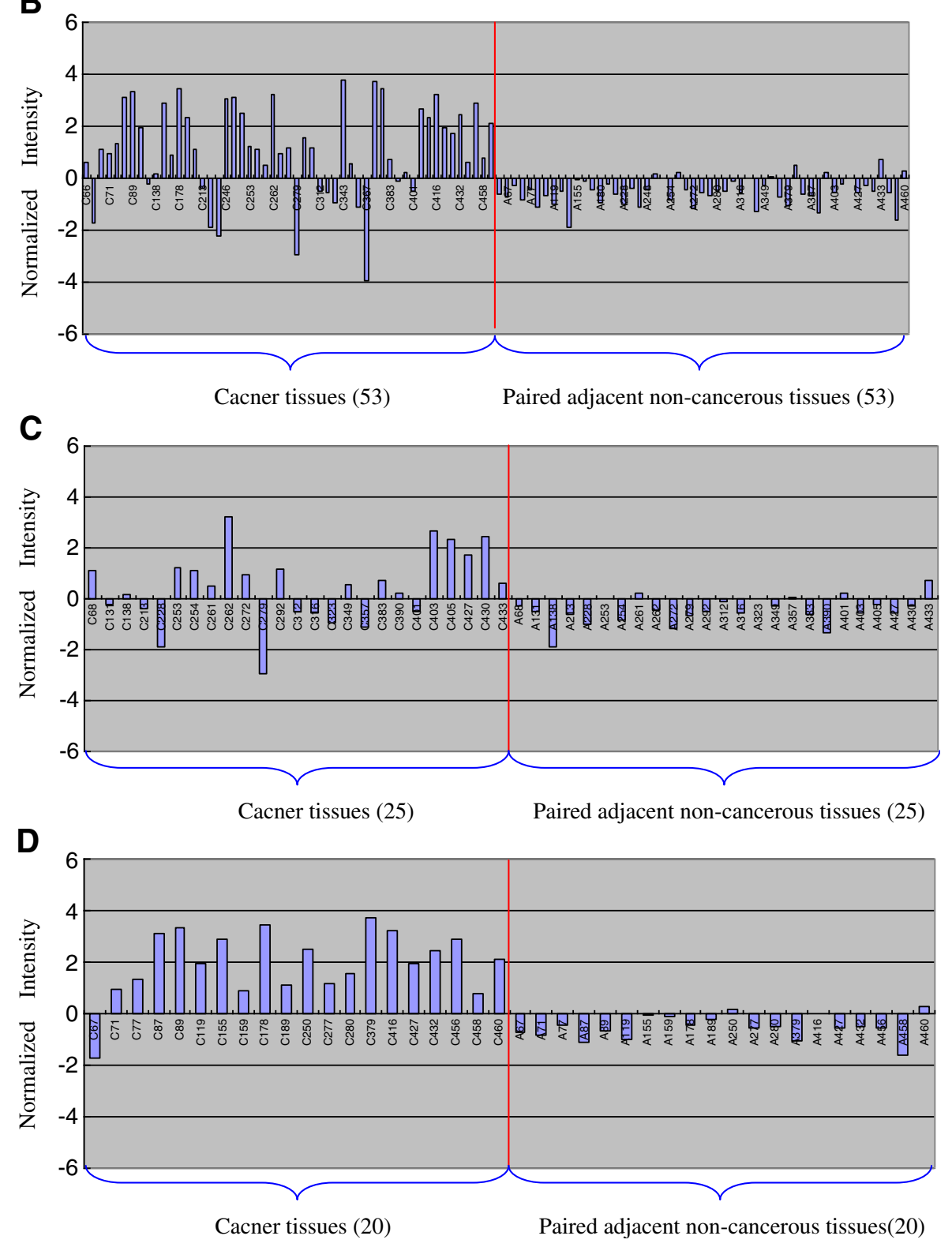

Figure 1 (See legend on next page.) 
(See figure on previous page.)

Figure $1 B C L 11 A$ mRNA is upregulated in cancer tissues compared to adjacent non-cancerous tissues. Normalized intensity of $B C L 11 A$ expression was determined by Affymetrix ${ }^{\circledast}$ mRNA array and compared between (A) all 114 NSCLC cancer tissues and 53 adjacent non-cancerous lung tissues; (B) 53 NSCLC cancer tissues and paired adjacent non-cancerous lung tissues; (C) 25 paired lung adenocarcinoma and adjacent lung tissues; (D) 20 paired lung squamous carcinoma and adjacent lung tissues. BCL11A was specifically upregulated in NSCLC tissues, especially in squamous cell lung carcinoma tissues.

\section{Predicted microRNAs targeting BCL11A were downregulated in NSCLC tissues}

MicroRNAs (miRNAs) have gained considerable attention as regulators of gene expression [10], and they play important roles in cellular differentiation and embryonic stem cell development [11]. We postulated that they may also play a role in modulating BCL11A expression. In order to test whether miRNAs play a role in controlling BCL11A expression, we computationally identified those that might contribute to BCL11A regulation. Since it has been generally accepted that intersecting the results of multiple prediction algorithms can increase specificity, we chose to intersect the results of the prediction programs PicTar (4-way), miRanda (miRBase), and TargetScan [12-14]. Overall, only three miRNAs were found by these programs to target BCL11A, including miR-1, miR-135a, and miR-30a (Figure 5A). We intersected these three miRNAs with results of microarray-based miRNA expression profiles (data not shown) and found that only miR-1 and miR-30a were downregulated in NSCLC tissues. We further analyzed the expression of these two microRNAs in 22 paired tissues of NSCLC patients by TaqMan real-time PCR. As compared to adjacent tissues, miR-30a and miR-1 were downregulated by 3.9 - and 12.1 -fold in cancer tissues, respectively (Figure 5B).

Table 1 Relationships between BCL11A expression and clinicopathological factors.

\begin{tabular}{|c|c|c|c|c|c|c|c|c|}
\hline \multirow[b]{2}{*}{ Parameter } & \multicolumn{4}{|c|}{ BCL11A mRNA expression $(n=114)$} & \multicolumn{4}{|c|}{ BCL11A protein expression $(n=113)$} \\
\hline & No. & Low (\%) & High (\%) & $P$-value & No. & Low (\%) & High (\%) & $P$-value \\
\hline Age & & & & 0.984 & & & & 0.325 \\
\hline$<60 \mathrm{yr}$ & 50 & $21(42.0)$ & $29(58.0)$ & & 70 & $44(62.9)$ & $26(37.1)$ & \\
\hline$\geq 60 \mathrm{yr}$ & 64 & $27(42.2)$ & $37(57.8)$ & & 43 & $23(53.5)$ & $20(46.5)$ & \\
\hline Sex & & & & 0.708 & & & & 0.133 \\
\hline Female & 33 & $13(39.4)$ & $20(60.6)$ & & 36 & $25(69.4)$ & $11(30.6)$ & \\
\hline Male & 81 & $35(43.2)$ & $46(56.8)$ & & 77 & $42(54.5)$ & $35(45.5)$ & \\
\hline Performance status & & & & 0.826 & & & & 0.399 \\
\hline 0 & 58 & $25(43.1)$ & $33(56.9)$ & & 56 & $31(55.4)$ & $25(44.6)$ & \\
\hline $1-2$ & 56 & $23(41.1)$ & $33(58.9)$ & & 57 & $36(63.2)$ & $21(36.8)$ & \\
\hline Smoking status & & & & 0.617 & & & & 0.003 \\
\hline No & 53 & 21 (39.6) & $32(60.4)$ & & 56 & $41(73.2)$ & $15(26.8)$ & \\
\hline Yes & 61 & $27(44.3)$ & $34(55.7)$ & & 57 & $26(45.6)$ & $31(54.4)$ & \\
\hline Histological type & & & & 0.921 & & & & 0.001 \\
\hline$A C$ & 68 & $29(42.6)$ & $39(57.4)$ & & 68 & $50(73.5)$ & $18(26.5)$ & \\
\hline SCC & 35 & $15(42.9)$ & $20(57.1)$ & & 36 & $12(33.3)$ & $24(66.7)$ & \\
\hline LCC & 11 & $4(36.4)$ & 7 (63.6) & & 9 & $5(55.6)$ & $4(44.4)$ & \\
\hline Tumor size & & & & 0.345 & & & & 0.124 \\
\hline$\leq 3.0 \mathrm{~cm}$ & 63 & $29(46.0)$ & $34(54.0)$ & & 59 & $39(66.1)$ & $20(33.9)$ & \\
\hline$>3.0 \mathrm{~cm}$ & 51 & 19 (37.3) & $32(62.7)$ & & 54 & $28(51.9)$ & $26(48.1)$ & \\
\hline Lymph node status & & & & 0.013 & & & & 0.023 \\
\hline No & 72 & $24(33.3)$ & $48(66.7)$ & & 72 & $37(51.4)$ & 35 (48.6) & \\
\hline $\mathrm{N} 1-2$ & 42 & $24(57.1)$ & $18(42.9)$ & & 41 & $30(73.2)$ & $11(26.8)$ & \\
\hline Pathological stage & & & & 0.040 & & & & 0.048 \\
\hline $\mid-\|$ & 89 & $33(37.1)$ & $56(62.9)$ & & 88 & $48(54.5)$ & $40(45.5)$ & \\
\hline III-IV & 25 & $15(60.0)$ & $10(40.0)$ & & 25 & $19(76.0)$ & $6(34.0)$ & \\
\hline
\end{tabular}




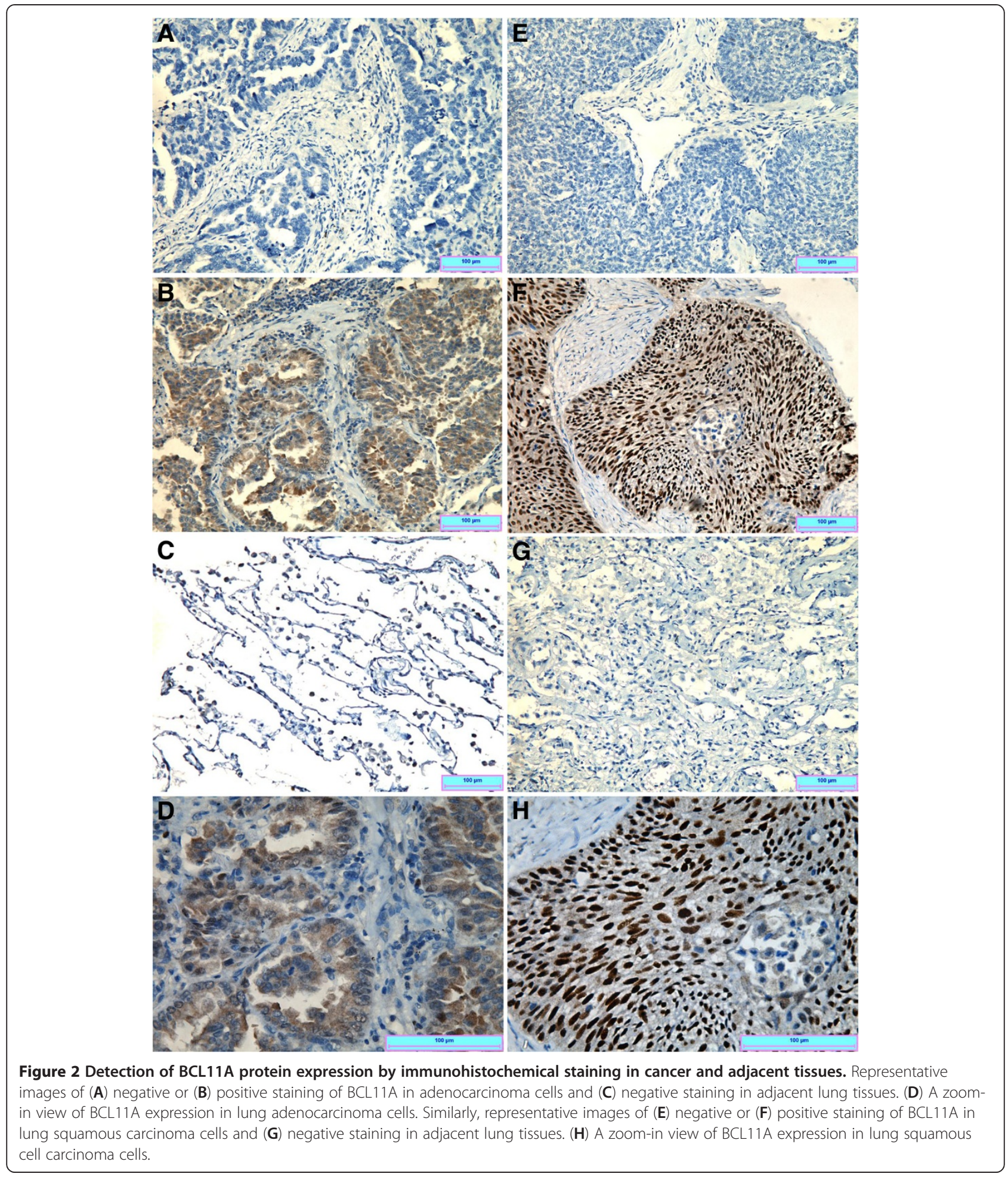

\section{BCL11A is the target of miR-30a}

After co-transfection of pmirGLO dual luciferase reporter plasmids carrying the 3 '-UTR sequence of BCL11A and plasmids expressing miR-30a, miR-1, or a control miRNA into A549 and NIH3T3 cells, firefly luciferase activity was normalized to Renilla luciferase activity. Significant repression was only seen in miR-30a-transfected cells, but not in either miR-1- or control miRNA-transfected cells. When the nucleotide acid sequence in the BCL11A 3' UTR that was complementary to the "seed region" of miR-30a was replaced, the repression effect was completely rescued (Figure 6A-C). 


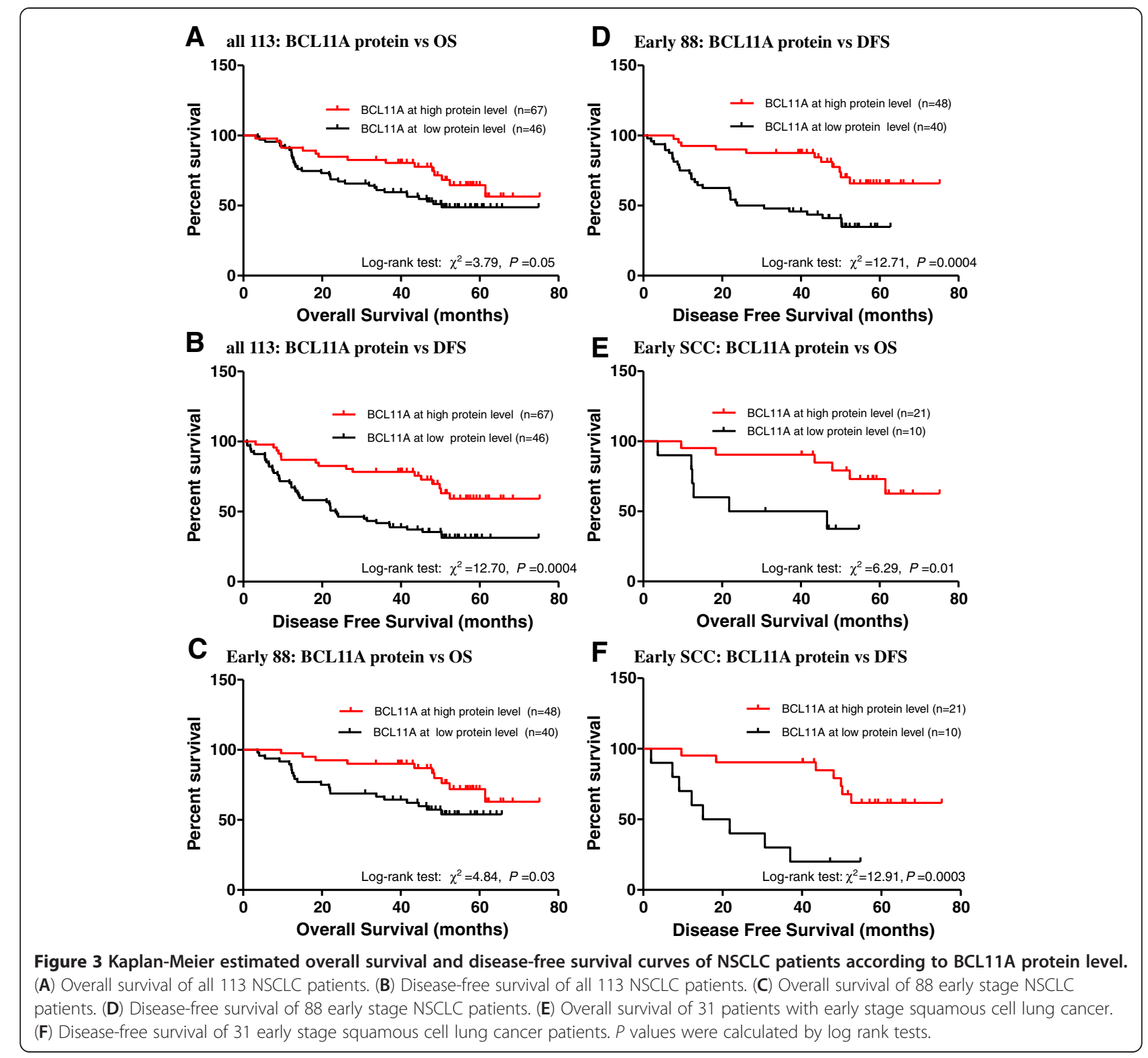

\section{BCL11A protein expression is inhibited by miR-30a} in vitro

Since the 3'UTR of BCL11A could be a target of miR30a, we tested whether 3'UTR binding by miR-30a resulted in changes in BCL11A protein expression in H460 and H520 cell lines. First, we performed an immunoblot analysis using a BCL11A-specific antibody. After transfection with miRNAs, BCL11A protein expression in the $\mathrm{H} 520$ cell line was repressed by miR-30a, but not by miR-1 or control miRNA (Figure 6D). After transient transfection with a plasmid expressing miR-30a, BCL11A staining in $\mathrm{H} 460$ cells was significantly downregulated compared to those transfected with control miRNA, as determined by immunofluorescence and confocal microscopy (Figure 6E).

\section{Discussion}

The current study investigated the role of the protooncogene BCL11A (B-cell lymphoma/leukemia 11A) in NSCLC. Our results demonstrated that BCL11A was upregulated in clinical NSCLC tissue samples at both transcriptional and translational levels. Statistical analyses demonstrated that BCL11A expression was associated strongly with lymph node status, disease stage, and the survival of patients with NSCLC. Moreover, we also observed some significant differences between BCL11A 
Table 2 Multivariate Cox regression analysis of BCL11A and survival in patients with early stage cancer (IA-IIB)

\begin{tabular}{|c|c|c|c|}
\hline Variable & $\begin{array}{c}\text { Hazards ratio } \\
(95 \% \mathrm{Cl})\end{array}$ & $\begin{array}{c}\text { Unfavorable / } \\
\text { favorable }\end{array}$ & $P$ value \\
\hline \multicolumn{4}{|l|}{ os } \\
\hline Age & $1.07(1.03-1.12)$ & $\geq 60 /<60$ & 0.002 \\
\hline Tumor size & $1.29(1.08-1.57)$ & $\geq 3 \mathrm{~cm} /<3 \mathrm{~cm}$ & 0.007 \\
\hline Lymph node status & $9.09(3.58-23.8)$ & $\begin{array}{l}\text { Positive/ } \\
\text { negative }\end{array}$ & 0.000 \\
\hline $\begin{array}{l}\text { Performance } \\
\text { status }\end{array}$ & $2.46(1.06-5.68)$ & $1-2 / 0$ & 0.036 \\
\hline BCL11A protein & $0.27(0.11-0.69)$ & Higher/lower & 0.006 \\
\hline Histological type & & & 0.003 \\
\hline SCC $^{\#}$ & $0.16(0.05-0.54)$ & SCC/AC & 0.003 \\
\hline $\operatorname{LCC}^{*}$ & $0.10(0.03-0.38)$ & LCC/AC & 0.001 \\
\hline \multicolumn{4}{|l|}{ DFS } \\
\hline Tumor size & $1.32(1.11-1.56)$ & $\geq 3 \mathrm{~cm} /<3 \mathrm{~cm}$ & 0.002 \\
\hline $\begin{array}{l}\text { Lymph node } \\
\text { status }\end{array}$ & $2.56(1.32-5.00)$ & $\begin{array}{l}\text { Positive/ } \\
\text { negative }\end{array}$ & 0.006 \\
\hline BCL11A protein & $0.25(0.12-0.51)$ & Higher/lower & 0.001 \\
\hline
\end{tabular}

protein expression and histologic origin. The survival analysis found that patients with higher BCL11A expression had better outcomes. BCL11A protein was an independent prognostic factor of disease-free survival and overall survival for early stage patients (IA-IIB), especially for early stage squamous carcinoma patients.

The proto-oncogene $B C L 11 A$ spans over $102 \mathrm{~kb}$ on chromosome 2 p16 and is implicated in several neoplasms $[9,15]$. Its sequence is phylogenetically conserved and codes for a zinc-finger protein with an unusual $\mathrm{C}_{2} \mathrm{HC}$ zinc finger at the $\mathrm{N}$-terminus and six other Krüppel-like $\mathrm{C}_{2} \mathrm{H}_{2}$ zinc fingers near the Cterminus [10]. BCL11A was reported to show restricted expression in fetal liver, bone marrow, lymphoid, and brain tissue $[16,17]$, but our results clearly demonstrated that the proto-oncogene $B C L 11 A$ was actively expressed in NSCLC. This phenomenon suggested that abnormal activation of the proto-oncogene BCL11A might contribute to tumorigenesis of NSCLC, although the details of its function have not yet to be clarified.

Table 3 Multivariate Cox regression analysis of BCL11A and survival in patients with early stage squamous carcinoma

\begin{tabular}{lccc}
\hline Variable & $\begin{array}{c}\text { Hazard ratio } \\
\mathbf{( 9 5 \% ~ C l )}\end{array}$ & $\begin{array}{c}\text { Unfavorable / } \\
\text { favorable }\end{array}$ & $\boldsymbol{P}$ value \\
\hline OS & $1.38(1.00-1.91)$ & $\geq 3 \mathrm{~cm} /<3 \mathrm{~cm}$ & 0.049 \\
\hline Tumor size & $0.18(0.05-0.66)$ & Higher/lower & 0.010 \\
\hline BCL11A protein & & & \\
\hline DFS & & Higher/lower & 0.001 \\
\hline BCL11A protein & $0.17(0.06-0.50)$ & &
\end{tabular}

BCL11A can bind to $5^{\prime}$-GGCCGG-3' DNA motifs with high affinity, and can promote the deacetylation of histone $\mathrm{H} 3 / \mathrm{H} 4$ associated with the promoter reigons of mammalian target genes, thereby causing transcriptional repression $[15,18]$. A previous study reported that enhanced BCL11A expression could repress P21 induction, which correlated with reduced colony formation and cell cycle arrest of leukemic cells [19]. Yin et al. also reported that $B C L 11 A$ acts as an oncogene and causes leukemia in the absence of NF1 in mice, perhaps through suppression of P21 induction and thus promotion of cell growth [20]. As is well known, the NF1 suppressor gene is also frequently inactivated in NSCLC [21]. Whether the same event occurs in NSCLC tumorigenesis remains unclear. Apart from transcriptional repression, $\mathrm{Yu}$ et al. found that $\mathrm{BCL11 \textrm {A }}$ can also upregulate expression of BCL2, BCL2-xL, and MDM2 which inhibits P53 activities [22]. These molecular alterations occur frequently in solid tumors including lung cancer. Therefore, BCL11A may contribute to tumorigenesis through effects on apoptosis, the cell cycle, and DNA damage repair, but the exact mechanisms warrant further investigation.

When compared to patients with advanced stage NSCLC and lymph node involvement, BCL11A expression was much higher in patients with early stage cancer and no lymph node involvement, which suggests that activation of the BCL11A proto-oncogene might be an early stage event in NSCLC. We performed a survival analysis and found that high BCL11A expression correlated with better survival and DFS, results similar to previous data on lymphoma. Pulford et al. performed immunolabelling studies on 107 cases of DLBCL (diffuse large B-cell lymphoma) and showed a trend towards poorer survival in patients with BCL11A-xL-negative tumors [23]. Recently, it was reported that BCL11A was amplified in lung squamous cell carcinoma (SCC), and this amplification was significantly more frequent in SCC samples of NSCLC patients without metastases [24]. In our study, BCL11A protein level was a protective factor for both DFS and OS of early stage NSCLC patients, especially for early stage SCC. Therefore, one wonders whether BCL11A inhibits cancer metastasis and acts as a tumor suppressor. Liu et al. found that transplantation of BCL11A-knockout murine fetal liver cells resulted in T-cell leukemia in recipient mice, suggesting that BCL11A may be a non-cell autonomous T-cell tumor suppressor gene [6]. Moreover, another member of the BCL11 family, BCL11B, has been suggested as a tumor suppressor gene, acting via an increase in resistance to DNA damage $[25,26]$. In addition to the potential mechanism mentioned above, two cell cycle checkpoint members, P21 and CHEK1, which contribute to several cell cycle checkpoints, including the G1/S and G2/ 

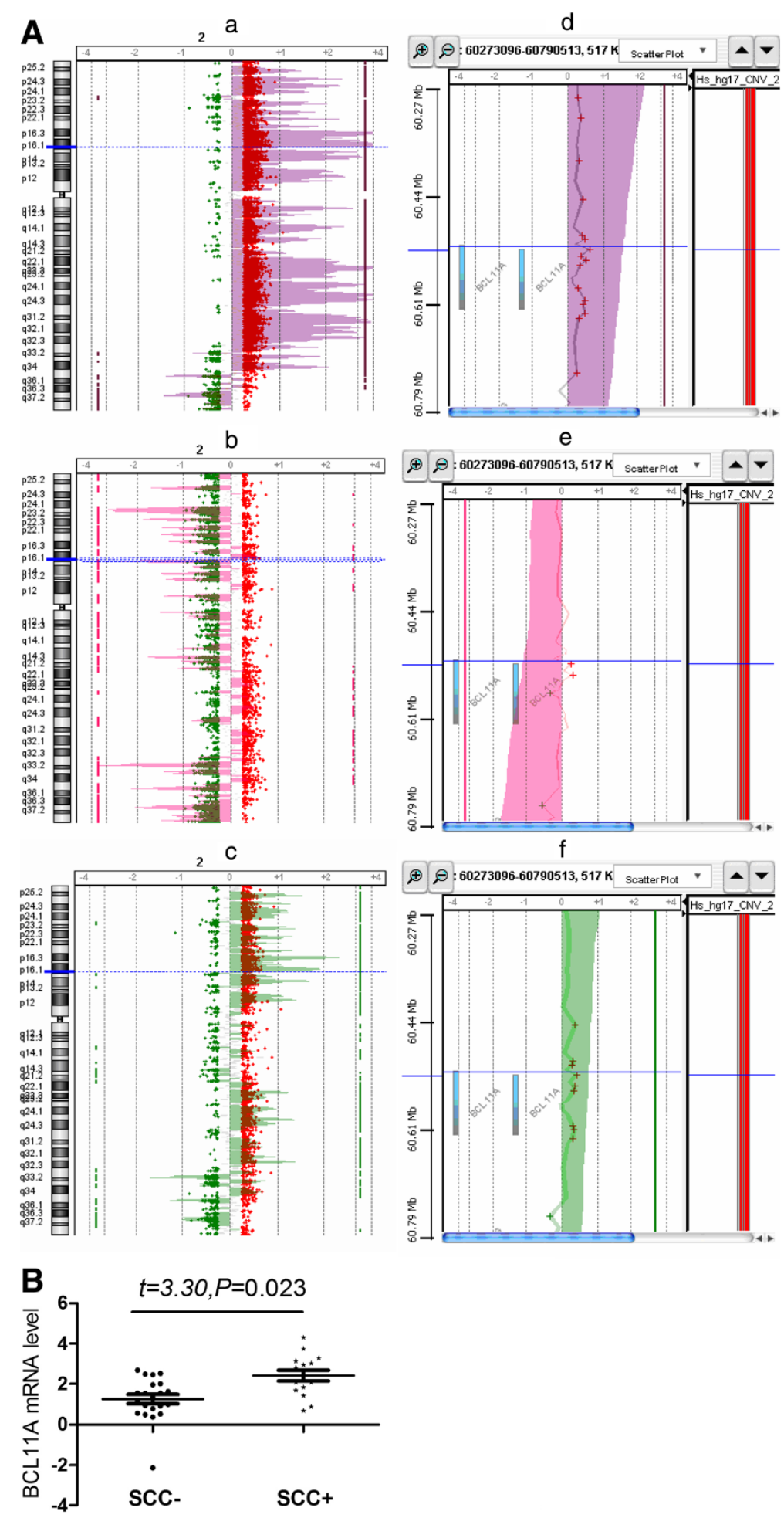

Figure $4 B C L 11 A$ gene amplification is frequently observed in squamous cell carcinoma. (A) Chromosome and gene views of copy number variance (CNV) signals in NSCLC with different histological origins. Log2 ratios of CNV probe intensity are plotted on the $x$ axis against genomic location on the $y$ axis. The blue horizontal line represents the location of the BCL11A gene on chromosome 2. $\mathbf{a}$, $\mathbf{b}$, and $\mathbf{c}$ represent the chromosome views of squamous cell carcinoma, adenocarcinoma, and large cell carcinoma, respectively. $\mathbf{d}$, e, and $\mathbf{f}$ represent the gene views of squamous cell carcinoma, adenocarcinoma, and large cell carcinoma, respectively, which show a zoom-in view of BCL11A copy number variation on the 2p16.1 band. (B) Expression levels were compared between SCC samples without high copy number amplification (SCC-) and SCC with high copy number amplification (SCC+) of the genomic region harboring the gene of interest. 


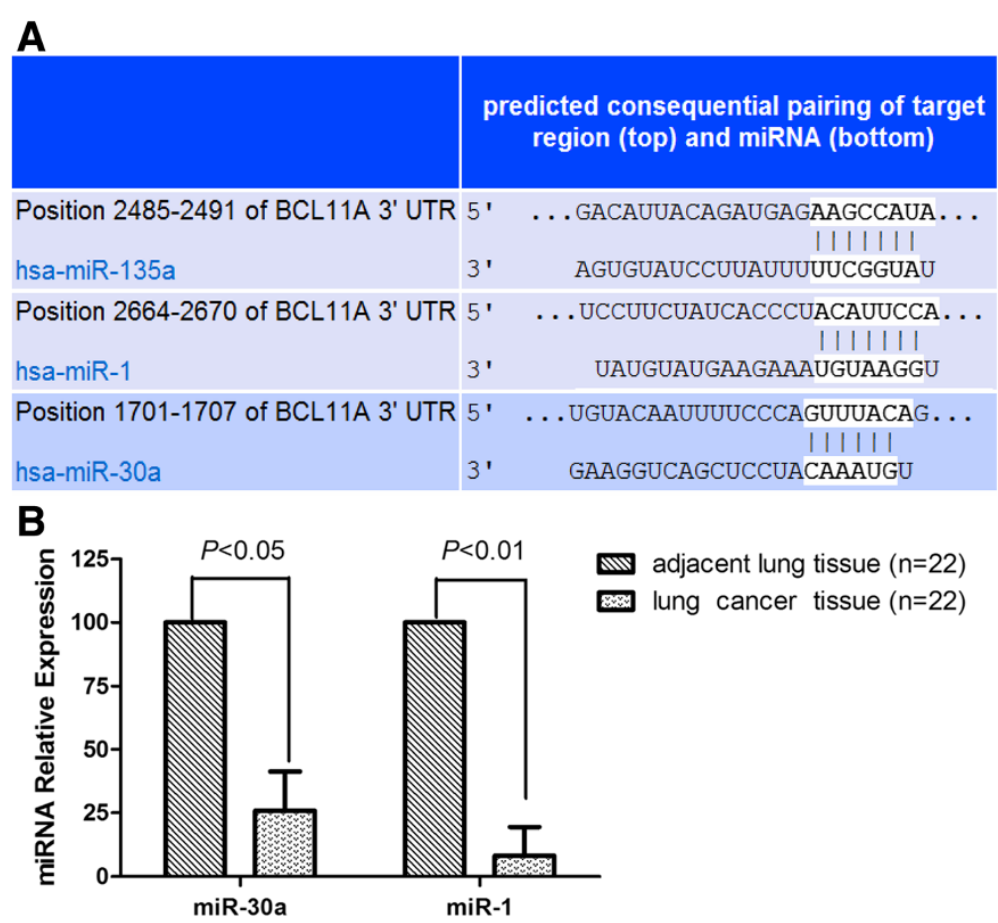

Figure 5 Computationally predicted miRNAs targeting BCL11A are downregulated in NSCLC tissues. (A) Schematic of three different binding sites in the BCL11A 3'UTR complementary to the "seed region" of three miRNAs, including miR-135a, miR-1, and miR-30a. (B) miR-1 and miR-30a were downregulated in NSCLC tissues compared to adjacent lung tissues $(n=22)$.

$M$ checkpoints might also account for this process. Activation of P21 and CHEK1 can promote DNA repair, maintain genomic integrity, and attenuate chemo- or radiotherapeutic efficacy $[27,28]$. Collective evidence suggests that P21 and CHEK1 could be targets of BCL11A. This interesting phenomenon implied BCL11A might have a dual role during NSCLC progression. In the initiation of tumorigenesis, BCL11A might act as an oncogene by repressing $\mathrm{P} 21, \mathrm{CHEK} 1$, and $\mathrm{P} 53$, resulting in genomic instability and contributing to carcinogenesis. However, once the tumor is established, BCL11A might inhibit cancer metastasis and improve the sensitivity of chemo- or radiotherapy, thereby contributing to better survival. This hypothesis should be further investigated in future studies.

There were several mechanisms that accounted for the abnormal activation of the proto-oncogene BCL11A reported in malignant hematological disease, including genomic amplification, chromosomal translocation, and retroviral integration. In this study, BCL11A gene copy number gains were frequently observed in squamous cell lung cancer patients, but not in adenocarcinoma or large cell carcinoma patients, which matched the results of Boelens et al. They observed that about $12 \%$ of squamous cell lung cancers had high copy number amplifications on chromosomal regions 2p15-p16.1, In addition, a significantly higher average expression level was observed for $B C L 11 A$ in the SCC samples with amplifications as compared to SCC without amplifications. Combined results demonstrated that genomic amplification is partially responsible for abnormal BCL11A expression in SCC tissues. MicroRNAs are approximately 21-nucleotide-long RNA regulators of gene expression at the translational level and control gene expression post-transcriptionally by regulating mRNA translation in the cytoplasm $[29,30]$. Using the miRGen database [14], we found that BCL11A is a common target of miR-1 and miR-30a, which were downregulated in NSCLC compared to normal lung tissues. Thus, we performed miRNA transfection to explore whether downregulation of miRNAs in NSCLC tissues contributed to deregulation of BCL11A. The results demonstrated that miR-30a could inhibit BCL11A protein expression in vitro. Thus, inactivation of miR-30a might also contribute to proto-oncogene BCL11A activation. However, whether these two mechanisms act synergistically remains unknown. In addition, we did not elucidate the biological functions of BCL11A and miR-30a in NSCLC.

In summary, we report here for the first time the potential role of BCL11A in diagnosing and predicting the prognosis of patients with NSCLC, especially those with early stage lung squamous carcinoma. BCL11A was highly and specifically expressed in cancer tissues rather than adjacent non-cancerous tissues. Deregulated expression of BCL11A might be partly caused by miR$30 \mathrm{a}$ inactivation and genomic BCL11A amplification. 

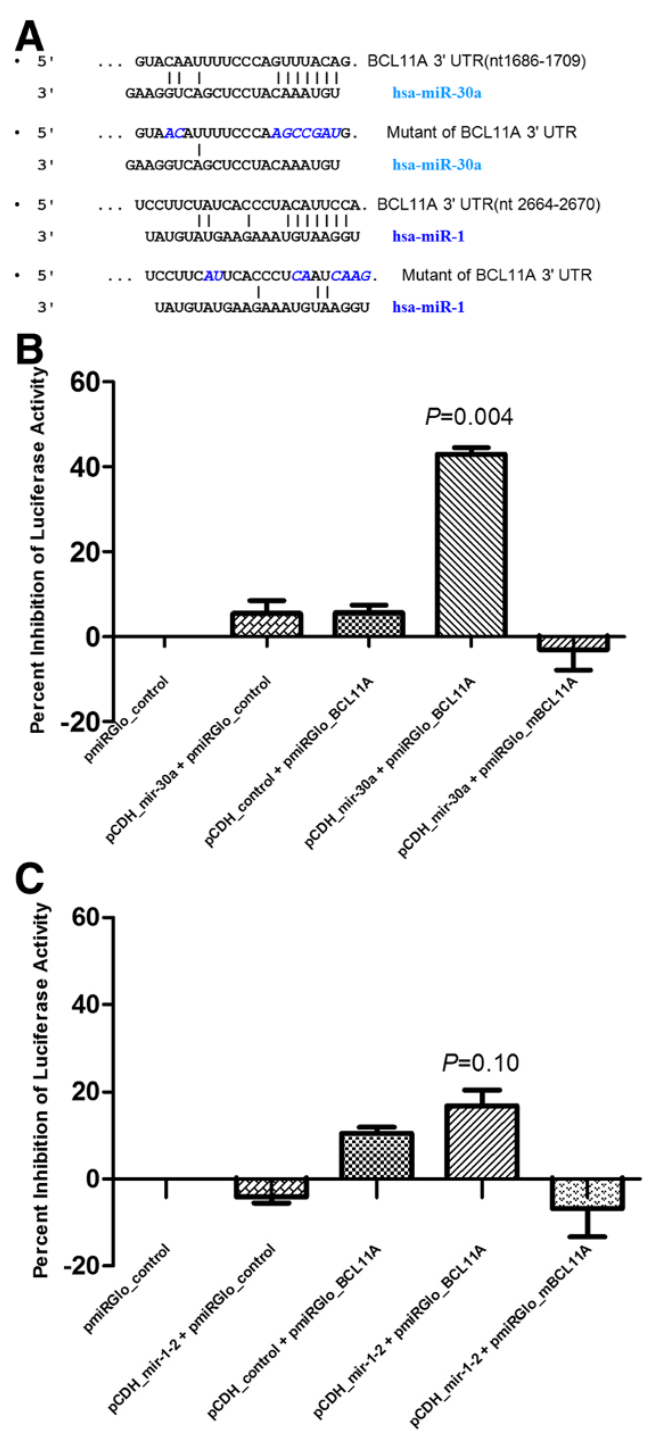

Figure 6 The BCL11A 3'-UTR is the target of miR-30a, but not of miR-1. pmirGLO-BCL11A luciferase constructs, containing a wild-type or mutated BCL11A 3'-UTR (A), were transfected into A549 cells with (B) miR-30a, (C) miR-1, or control miRNA vectors. Relative repression of firefly luciferase expression was standardized to Renilla luciferase activity. The reporter assays were performed three times with essentially identical results. The $P$ value was calculated comparing control microRNA and miR-30a or miR-1. (D) miR-30a downregulated BCL11A protein expression in H520 cells. Control miRNAs were also used for transfection. The experiment was performed three independent times, and representative results are displayed. CD511B represents the PCDH-CMV-MCS-EF1-CopGFP empty vector. (E) Immunofluorescence staining intensity of BCL11A protein in H460 cells observed by confocal microscopy after transfection with miR-30a or control miRNA. Magnification, $\times 200$.

Thus, future studies should assess whether BCL11A functions as an oncogene or tumor suppressor gene in NSCLC, whether BCL11A can be a better diagnostic and prognostic biomarker of early stage squamous cell lung cancer.

\section{Conclusions}

Activation of the proto-oncogene $B C L 11 A$ may be a potential diagnostic and prognostic biomarker of NSCLC, and is modulated by miR-30a and gene amplification.

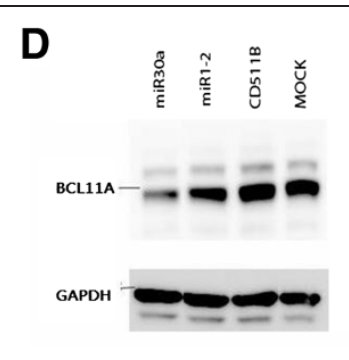

E

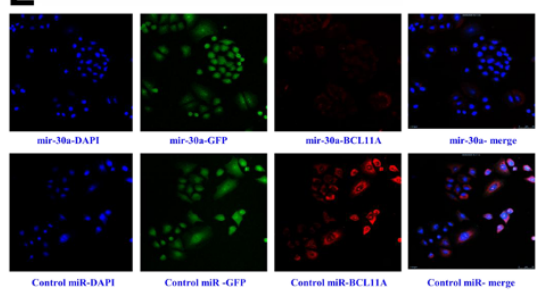


was performed according to standard follow-up protocol. The median follow-up period was 47.2 months (range, 3.1-75.2 months). Hemotoxylin and eosin (H\&E) staining was performed on sections of each tissue to determine the percentage of tumor cells by two independent pathologists. Only those samples with tumor content $\geq 80 \%$ were allowed to enter this study. Demographic and clinical characteristics of the patients are listed in Additional file 2: Table S1.

\section{Cell lines}

The NIH3T3, A549,NCI-H460 and H520 cell lines were obtained from the American Type Culture Collection (Manassas, VA, USA) and were cultured in RPMI 1640 medium (Invitrogen, Carlsbad, CA) supplemented with $10 \%$ fetal bovine serum (Thermo, Waltham, MA). Cells were maintained at $37^{\circ} \mathrm{C}$ in a humidified $5 \% \mathrm{CO}_{2}$ incubator (Thermo).

\section{Gene expression profiling using Affymetrix GeneChip ${ }^{\circledR}$ technology}

Cancer tissue samples from 114 cases and 53 adjacent non-tumor tissue samples were subject to gene profiling tests using the Affymetrix human genome U133 plus 2.0 GeneChip (Affymetrix, Santa Clara, CA, USA). Agilent GeneSpring ${ }^{\ominus}$ software (Agilent Technologies, Santa Clara, CA, USA) was utilized for the analysis of differentially expressed genes between cancerous and adjacent non-cancerous tissues with a Bonferroni correction FDR (false discovery rate) of 0.0001. Normalized intensities of Affymetrix probe sets targeting BCL11A (222891_s_at, 219497_s_at, 219498_s_at, 1559078_s_at, and 210347_s_at) were extracted and averaged for plotting across all samples.

\section{Immunohistochemistry on tissue microarrays}

Tissue microarrays were constructed using a manually operated tissue arrayer (Beecher Instruments, Sun Prairie, WI, USA). A total of 113 formaldehyde-fixed paraffinembedded (FFPE) samples were successfully used to generate four serial tissue arrays. Immunohistochemical staining process was performed according to the protocol provided by DAKO (DakoCytomation, Glostrup, Denmark) [31]. A primary mouse monoclonal antibody against human BCL11A (ab19487; Abcam, Cambridge, USA) was applied to the sections at a dilution of 1:200 for $1 \mathrm{~h}$ at room temperature. The sections were counterstained with Harris's hematoxylin. Each tumor was assigned a score according to the intensity of the nucleic or cytoplasmic staining $(0=$ no staining, $1=$ weak staining, $2=$ moderate staining, and $3=$ strong staining) and the proportion of stained tumor cells $(0=0 \%, 1=1-10 \%, 2=11-50 \%, 3=$ $51-80 \%$, and $4=81-100 \%$ ) [31], as judged by two pathologists, independently. The final immunoreactive score was determined by multiplying the intensity scores by the extent of positivity scores of stained cells, with a minimum score of 0 and a maximum score of 12 . Tumors with scores $\geq 6$ were classified into the high BCL11A expression group, while the others were classified into the low BCL11A expression group.

\section{Array-based comparative genome hybridization (aCGH) analysis}

Array-CGH tests were performed using the Agilent $\mathrm{Hu}$ man Genome Microarray kit 244A (Agilent Technologies). Labeling and hybridization were performed according to the protocol provided by Agilent (Protocol v4.0, June 2006). Ten probes targeting BCL11A on the Agilent aCGH chip (A_16_P15674055, A_16_P15674092, A_16_ P00386506, A_14_P202460, A_16_P15674199, A_14_P12 9011, A_16_P15674243, A_16_P00386603, A_16_P0038 6649, and A_14_P106886) were used to determine the normalized logarithmic intensity of BCL11A copy number changes. Log intensities $>0.30$ were considered to indicate gene amplification.

\section{BCL11A-targeting microRNA prediction}

To identify miRNAs targeting BCL11A, we integrated the output results from the DIANA miRGen online database, which were generated using multiple prediction programs: TargetScan [http://www.targetscan.org/], PicTar [http://www.pictar.org/], miRanda [http://www. microrna.org/microrna/] and DIANA microT [http:// diana.pcbi.upenn.edu/cgi-bin/micro_t.cgi] [14]. We integrated the results for each prediction tool and exported the sequences of predicted binding sites for each microRNA in the BCL11A 3'UTR.

\section{Quantification of microRNA expression using real time qRT-PCR}

Twenty-two paired NSCLC tissues undergoing microRNA analyses included (nine cases of adenocarcinoma, eight of squamous cell carcinoma, and five of large cell carcinoma) were subjected to microRNA analyses. Quantitative RTPCR amplification of microRNA and RNU6 was performed using the pre-developed Assay-on-Demand Gene Expression Set for the microRNA (Applied Biosystems, Foster City, CA). Briefly, 10 ng of total RNA was reverse transcribed into cDNA using High Capacity cDNA sysnthesis kit (Applied Biosystems) in the presence of stem-loop primers. All reactions were performed in triplicates using $20 \mu$ samples containing $1 \mathrm{ng}$ of cDNA. The qRT-PCR reaction was performed using in an ABI PRISM 7500FAST system (Applied Biosystems), with the following cycling conditions: $10 \mathrm{~min}$ at $95^{\circ} \mathrm{C}$, and 40 cycles of $95^{\circ} \mathrm{C}$ for $15 \mathrm{~s}$ and $60^{\circ} \mathrm{C}$ for $60 \mathrm{~s}$. microRNA expression was quantified using the comparative CT (cycle at threshold) method, which normalizes the $\mathrm{CT}$ values to an internal 
housekeeping ncRNA (RNU6) and calculates the relative expression value [32].

\section{MicroRNA vectors and luciferase reporter assays}

Two microRNA-expressing plasmids were constructed. DNA fragments carrying pre-microRNAs were amplified by high-fidelity PCR using genomic DNA from a healthy blood donor as a template. PCR reactions were performed using the high-fidelity Phusion polymerase (New England Biolabs), and specific primers ending with sequences for EcoRI and NotI enzymes (New England Biolabs) (Additional file 3: Table S2). The amplified fragment was enzyme digested and subsequently cloned into pCDH-CMV-MCS-EF1-copGFP (System Biosciences, Mountain View, CA) at the EcoRI and NotI sites. Expression of the mature microRNAs was verified by TaqMan real-time qRT-PCR. Luciferase constructs were created by ligating oligonucleotides (Additional file 4: Table S3) containing the wild type or mutant target site of the BCL11A 3-UTR into the DraI and XbaI sites of the pmirGLO vector (Promega, Madison, WI). Vectors were sequenced using an $E B V$ reverse primer: 5'-GTGGTTT GTCCAAACTCATC-3' . A549 and NIH3T3 cells (low endogenous BCL11A expression; data not shown) were cotransfected with $0.5 \mu \mathrm{g}$ of pmirGLO luciferase reporter vector containing a wild type or mutant target site and $0.5 \mu \mathrm{g}$ of the microRNA plasmid using Lipofectamine ${ }^{\mathrm{mm}}$ LTX reagent (Invitrogen, Carlsbad) in 12-well plates. After incubation for $48 \mathrm{~h}$, the cells were lysed and luciferase assays were conducted using a GloMax ${ }^{\mathrm{Tm}}$ 20/20 Luminometer (Promega). Firefly luciferase activity was normalized to Renilla luciferase activity. Each experiment was performed in triplicate [32].

\section{Western blotting}

H520 cells (high endogenous BCL11A expression; data not shown) were transfected with the control microRNAor miR-30a-expressing vector. Approximately $48 \mathrm{~h}$ after transfection, the cells were lysed. Western blotting was performed as described previously [33]. The anti-BCL11A antibody (ab19487) was obtained from Abcam and was used at a dilution of 1:1,000.

\section{Visualization of intracellular BCL11A protein expression by confocal microscopy}

Indirect immunofluorescence staining was used to determine BCL11A expression in miR-30a-transfected cells as described previously [34]. Briefly, H460 cells (high endogenous BCL11A expression; data not shown) were transfected with the control microRNA- or miR-30a-expressing vector, and $48 \mathrm{~h}$ after transfection, the cells were fixed with cold methanol. A primary antibody against BCL11A (ab19487; Abcam) was applied at a dilution of 1:100, followed by a secondary antibody conjugated to Alexa Fluor 635 (Invitrogen). DAPI (Invitrogen) was used as a nuclear stain, and GFP was used to monitor transfection efficiency.

\section{Statistical analysis}

Statistical significance was determined by paired or unpaired Student's $t$-tests in cases of standardized expression data. One-way analysis of variance (ANOVA) was performed for comparisons of multiple groups using the GraphPad Prism Software 5.01 (GraphPad, La Jolla, CA). Wilcoxon matched-pair tests and Mann-Whitney U tests were used for nonparametric analysis of non-Gaussian data. Error bars represent standard deviations ( \pm SD). Chi-square tests and McNemar's tests were used to assess the association of BCL11A level with clinical variables. Survival curves between subgroups divided according to BCL11A expression level were drawn using the Kaplan-Meier method, and significant differences among subgroups were compared by log-rank test. A multivariate analysis was performed using the stepwise method. Hazards ratios and 95\% confidence intervals were calculated using Cox proportional hazards models. $P$ values $<0.05$ were considered statistically significant.

\section{Additional files}

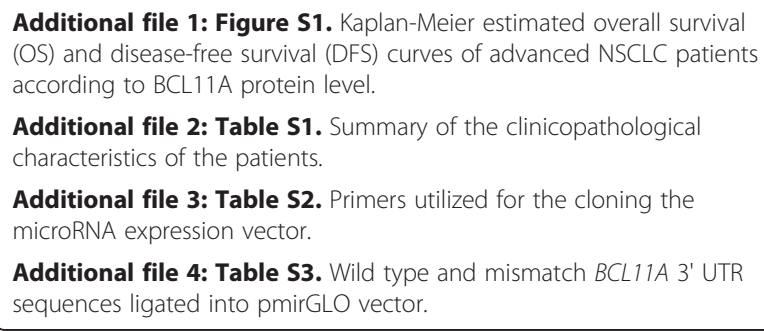

Additional file 3: Table S2. Primers utilized for the cloning the microRNA expression vector.

Additional file 4: Table S3. Wild type and mismatch BCL11A 3' UTR sequences ligated into PmirGLO vector.

\section{Competing interests}

The author(s) indicated no potential conflicts of interest.

\section{Authors' contributions}

BJ carried out the microRNA studies, data analysis and drafted the manuscript. $X Z$ performed the molecular genetic studies and participated in the manuscript writing. $X Y, J Y, Q Z, Z C$ provided study material or patients and assembled data. JS, WM, ZC, ZX, SC carried out vector construction, realtime PCR,and immunohistochemistry. YW conceived the study, participated in its design, and coordination and helped to draft the manuscript. All authors read and approved the final manuscript.

\section{Acknowledgements}

We thank Yi Gu, Xuhua Zhang, and Xiaolin Zhang of the AstraZeneca Innovation Center China for assistance with experiments. This work was supported by the grants from the National Natural Science Foundation of China (no.30772531 and no. 81071699), Guangdong Social Development of Science and Technology Plan Project (no.2011A030400010), and the Guangzhou Science and Technology Plan Project (no. 11BppZXaa6040020). This work was also supported by the joint laboratory of Guangdong General Hospital -AstraZeneca Innovation Center China. 


\section{Note}

The English in this document has been checked by at least two professional editors, both native speakers of English. For a certificate, please see: http:// www.textcheck.com/certificate/index/qhP4zS

\section{Author details}

${ }^{1}$ Guangdong Lung Cancer Institute, 106 Zhongshan Er Rd, Guangzhou 510080, China. ${ }^{2}$ Medical Research Center of Guangdong General Hospital, Guangdong Academy of Medical Sciences, 106 Zhongshan Er Rd, Guangzhou 510080, China. ${ }^{3}$ Graduate School of Southern Medical University, Guangzhou 510515, China.

Received: 18 February 2013 Accepted: 4 June 2013

Published: 12 June 2013

\section{References}

1. Herbst RS, Heymach JV, Lippman SM: Lung cancer. N Engl J Med 2008 359:1367-1380.

2. $\mathrm{Wu} Y \mathrm{~L}$, Zhou Q: Clinical trials and biomarker research on lung cancer in China. Expert Opin Ther Targets 2012, 16(Suppl 1):S45-S50.

3. Zhou Q, Zhang XC, Chen ZH, Yin XL, Yang JJ, Xu CR, Yan HH, Chen HJ, Su J, Zhong WZ, et al: Relative abundance of EGFR mutations predicts benefit from gefitinib treatment for advanced non-small-cell lung cancer. J Clin Oncol 2011, 29:3316-3321.

4. Liao WT, Wang X, Xu LH, Kong QL, Yu CP, Li MZ, Shi L, Zeng MS, Song LB: Centromere protein $\mathrm{H}$ is a novel prognostic marker for human nonsmall cell lung cancer progression and overall patient survival. Cancer 2009, 115:1507-1517.

5. Parsels LA, Qian Y, Tanska DM, Gross M, Zhao L, Hassan MC, Arumugarajah S, Parsels JD, Hylander-Gans L, Simeone DM, et al: Assessment of chk1 phosphorylation as a pharmacodynamic biomarker of chk1 inhibition. Clin Canc Res 2011, 17:3706-3715.

6. Liu P, Keller JR, Ortiz M, Tessarollo L, Rachel RA, Nakamura T, Jenkins NA, Copeland NG: Bcl11a is essential for normal lymphoid development. Nat Immunol 2003, 4:525-532.

7. Sankaran VG, Xu J, Ragoczy T, Ippolito GC, Walkley CR, Maika SD, Fujiwara Y, Ito $M$, Groudine M, Bender MA, et al: Developmental and speciesdivergent globin switching are driven by BCL11A. Nature 2009, 460:1093-1097.

8. Martin-Subero Jl, Gesk S, Harder L, Sonoki T, Tucker PW, Schlegelberger B, Grote W, Novo FJ, Calasanz MJ, Hansmann ML, et al: Recurrent involvement of the REL and BCL11A loci in classical Hodgkin lymphoma. Blood 2002, 99:1474-1477.

9. Satterwhite E, Sonoki T, Willis TG, Harder L, Nowak R, Arriola EL, Liu H, Price HP, Gesk S, Steinemann D, et al: The BCL11 gene family: involvement of BCL11A in lymphoid malignancies. Blood 2001, 98:3413-3420.

10. Avram D, Fields A, Senawong T, Topark-Ngarm A, Leid M: COUP-TF (chicken ovalbumin upstream promoter transcription factor)-interacting protein 1 (CTIP1) is a sequence-specific DNA binding protein. Biochem $J$ 2002, 368:555-563.

11. Marson A, Levine SS, Cole MF, Frampton GM, Brambrink T, Johnstone $S$, Guenther MG, Johnston WK, Wernig M, Newman J, et al: Connecting microRNA genes to the core transcriptional regulatory circuitry of embryonic stem cells. Cell 2008, 134:521-533.

12. Krek A, Grun D, Poy MN, Wolf R, Rosenberg L, Epstein EJ, MacMenamin P, da Piedade I, Gunsalus KC, Stoffel M, Rajewsky N: Combinatorial microRNA target predictions. Nat Genet 2005, 37:495-500.

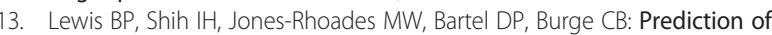
mammalian microRNA targets. Cell 2003, 115:787-798.

14. Megraw M, Sethupathy P, Corda B, Hatzigeorgiou AG: miRGen: a database for the study of animal microRNA genomic organization and function. Nucleic Acids Res 2007, 35:D149-D155.

15. Chen Z, Luo HY, Steinberg MH, Chui DH: BCL11A represses HBG transcription in K562 cells. Blood Cells Mol Dis 2009, 42:144-149.

16. Kuo TY, Hsueh YP: Expression of zinc finger transcription factor Bcl11A/ Evi9/CTIP1 in rat brain. J Neurosci Res 2007, 85:1628-1636.

17. Sankaran VG, Menne TF, Xu J, Akie TE, Lettre G, Van Handel B, Mikkola HK, Hirschhorn JN, Cantor AB, Orkin SH: Human fetal hemoglobin expression is regulated by the developmental stage-specific repressor BCL11A. science 2008, 322:1839-1842
18. Liu H, Ippolito GC, Wall JK, Niu T, Probst L, Lee BS, Pulford K, Banham AH, Stockwin L, Shaffer AL, et al: Functional studies of BCL11A: characterization of the conserved BCL11A-XL splice variant and its interaction with BCL6 in nuclear paraspeckles of germinal center B cells. Mol Canc 2006, 5:18.

19. Schmelz K, Wagner M, Dorken B, Tamm I: 5-Aza-2'-deoxycytidine induces p21WAF expression by demethylation of p73 leading to p53-independent apoptosis in myeloid leukemia. Int J Canc 2005, 114:683-695.

20. Yin B, Delwel R, Valk PJ, Wallace MR, Loh ML, Shannon KM, Largaespada DA: $A$ retroviral mutagenesis screen reveals strong cooperation between $\mathrm{Bcl11a}$ overexpression and loss of the Nf1 tumor suppressor gene. Blood 2009, 113:1075-1085.

21. Ding L, Getz G, Wheeler DA, Mardis ER, McLellan MD, Cibulskis K, Sougnez C, Greulich H, Muzny DM, Morgan MB, et al: Somatic mutations affect key pathways in lung adenocarcinoma. Nature 2008, 455:1069-1075.

22. Yu Y, Wang J, Khaled W, Burke S, Li P, Chen X, Yang W, Jenkins NA, Copeland NG, Zhang S, Liu P: Bcl11a is essential for lymphoid development and negatively regulates p53. J Exp Med 2012, 209:2467-2483.

23. Pulford K, Banham AH, Lyne L, Jones M, Ippolito GC, Liu H, Tucker PW, Roncador G, Lucas E, Ashe S, et al: The BCL11AXL transcription factor: its distribution in normal and malignant tissues and use as a marker for plasmacytoid dendritic cells. Leukemia 2006, 20:1439-1441.

24. Boelens MC, Kok K, van der Vlies P, van der Vries G, Sietsma H, Timens W, Postma DS, Groen HJ, van den Berg A: Genomic aberrations in squamous cell lung carcinoma related to lymph node or distant metastasis. Lung Canc 2009, 66:372-378.

25. Kamimura K, Ohi H, Kubota T, Okazuka K, Yoshikai Y, Wakabayashi Y, Aoyagi $Y$, Mishima $Y$, Kominami R: Haploinsufficiency of Bcl11b for suppression of lymphomagenesis and thymocyte development. Biochem Biophys Res Commun 2007, 355:538-542.

26. Kamimura K, Mishima Y, Obata M, Endo T, Aoyagi Y, Kominami R: Lack of $\mathrm{Bcl} 11 \mathrm{~b}$ tumor suppressor results in vulnerability to DNA replication stress and damages. Oncogene 2007, 26:5840-5850.

27. Dai $Y$, Grant S: New insights into checkpoint kinase 1 in the DNA damage response signaling network. Clin Canc Res 2010, 16:376-383.

28. Insinga A, Cicalese A, Faretta M, Gallo B, Albano L, Ronzoni S, Furia L, Viale A, Pelicci PG: DNA damage in stem cells activates p21, inhibits p53, and induces symmetric self-renewing divisions. Proc Natl Acad Sci USA 2013, 110:3931-3936.

29. Esquela-Kerscher A, Trang P, Wiggins JF, Patrawala L, Cheng A, Ford L, Weidhaas JB, Brown D, Bader AG, Slack FJ: The let-7 microRNA reduces tumor growth in mouse models of lung cancer. Cell Cycle 2008, 7:759-764.

30. Filipowicz W, Bhattacharyya SN, Sonenberg N: Mechanisms of posttranscriptional regulation by microRNAs: are the answers in sight? Nat Rev Genet 2008, 9:102-114.

31. Koo CL, Kok LF, Lee MY, Wu TS, Cheng YW, Hsu JD, Ruan A, Chao KC, Han CP: Scoring mechanisms of p16INK4a immunohistochemistry based on either independent nucleic stain or mixed cytoplasmic with nucleic expression can significantly signal to distinguish between endocervical and endometrial adenocarcinomas in a tissue microarray study. J Trans/ Med 2009, 7:25

32. Varambally S, Cao Q, Mani RS, Shankar S, Wang X, Ateeq B, Laxman B, Cao X, Jing X, Ramnarayanan K, et al: Genomic loss of microRNA-101 leads to overexpression of histone methyltransferase $\mathrm{EZH} 2$ in cancer. Science 2008, 322:1695-1699.

33. Wilber A, Nienhuis AW, Persons DA: Transcriptional regulation of fetal to adult hemoglobin switching: new therapeutic opportunities. Blood 2011, 117:3945-3953.

34. Reddy SD, Pakala SB, Ohshiro K, Rayala SK, Kumar R: MicroRNA-661, a C/EBPalpha target, inhibits metastatic tumor antigen 1 and regulates its functions. Canc Res 2009, 69:5639-5642.

doi:10.1186/1476-4598-12-61

Cite this article as: Jiang et al:: BCL11A overexpression predicts survival and relapse in non-small cell lung cancer and is modulated by microRNA-30a and gene amplification. Molecular Cancer 2013 12:61. 